ISSN 1112-9867

\title{
FASCICLE BEHAVIOUR ANALYSIS DURING FORWARD LUNGE EXERCISE: THE COMPARISONS BETWEEN TRAINING LOADS
}

\author{
A. M. Nadzalan ${ }^{1}$, N. I. Mohamad ${ }^{1}$, J. F. L. Low ${ }^{1}$, R. Ahmad ${ }^{1}$, \& E. Waqqash ${ }^{2}$ \\ ${ }^{1}$ Faculty of Sports Science and Coaching, Universiti Pendidikan Sultan Idris, Tanjung Malim, \\ Perak, Malaysia \\ ${ }^{2}$ Sports Centre, Universiti Malaya, Kuala Lumpur, Malaysia
}

Published online: 10 November 2017

\begin{abstract}
This study was conducted to determine and compare the fascicle behaviour during forward lunge exercise with different loadings lifted. Thirty recreationally active, untrained men (mean age $=21 \pm 0.83$ years old) were recruited and were assigned to perform forward lunge with 30\% 1RM (30FL) and 70\% 1RM (70FL) with both their dominant and non-dominant leg. For both dominant and non-dominant leg, results showed that FLmax, FLmin, PAmax and PAmin were all greater during 70FL compared to 30FL, $\mathrm{p}<0.001$. However, lengthening and shortening velocity were found to be greater during $30 \mathrm{FL}$ compared to $70 \mathrm{FL}, \mathrm{p}<0.05$. During both $30 \mathrm{FL}$ and $70 \mathrm{FL}$, all the fascicle behaviour variables were found to be greater in the dominant limb compared to non-dominant limb. In conclusion, fascicles were shown to response differently across different loadings executed that might affect the muscle architecture adaptations.
\end{abstract}

Keywords: training loadings, hypertrophy, muscle adaptation, asymmetry, specificity

Author Correspondence, e-mail: ali.nadzalan@fsskj.upsi.edu.my

doi: http://dx.doi.org/10.4314/jfas.v9i6s.80 


\section{INTRODUCTION}

Muscle architecture was found to be correlated with performance in several movements and sports performance [1-4]. In order to obtain the desired muscle architecture, training should be well planned especially the exercise selection and protocols as differences in these training variables could determine the adaptation occurred [5-7].

The architectures of skeletal muscles have the ability to adapt their size and length-force characteristics in order to meet functional demands in daily life [8]. One of the factors that can affect how the architectures adapt is the mechanical response and demands as a result of movement performed. Research on the fascicle behaviour has been conducted on resistance exercise [9-15] and walking and running [16-19].

It is our interest to study the fascicle behaviour during an exercise that is specific to movement in sports. According to the principle of specificity, exercise selection for training must be matched to the needs of the sporting activity to improve the functionality in the body parts the sport uses.

One of the most performed movements in sports is the forward lunge. Forward lunge started with a front step followed by a backward push. In order to enhance its effectiveness, the forward lunge should be performed with the lead leg been brought as far as possible to the front as in descent phase, the knee should not exceed the toe.

Given the relevance of the lunge pattern to sport and the necessity of the strength and conditioning specialist to load the movement pattern to enhance performance, lunge training could be one of the most specific training. However, in order to achieve desired outcomes, the lunge training could be adjusted as different lunge training have also demonstrated different adaptations $[5,20]$. The inclusion of lunge as training exercises should be beneficial as it will allow athletes or individuals to train and improve their ability for the movement and as a way to overload the athletes or individuals, various methods of lunge could be implemented during training sessions [21]. This includes manipulating the loadings lifted during the exercise.

The objective of this study is to determine and compare the effects of different loadings used during forward lunge exercise on the fascicle behaviour. The finding of this study is expected to enhance knowledge on the movement response during lunge exercise as one of the major movement performed in sports. 


\section{METHODOLOGY}

\subsection{Participants}

This study involved thirty (30) recreationally active, resistance untrained men volunteered to participate in this study. All the participants selected were males aged between 20-25 years old based on their year of birth. Participants had no medical problems and not consuming any performance enhancing supplementation. Participants were screened prior to testing using PAR-Q. Each participant read and signed an informed consent for testing and training approved by Universiti Pendidikan Sultan Idris.

\subsection{Procedures}

B-mode ultrasonography (F37, Aloka, Ltd, Tokyo, Japan) were used to obtain images of the VL fascicles for determination of fascicle length and fascicle angle throughout the movement. A $6 \mathrm{~cm}$ linear array, 13-MHz T-head transducer (UST 5413, Aloka Ltd, Tokyo, Japan) were used to collect images at $96 \mathrm{~Hz}$. The transducer were placed at $50 \%$ of the distance between the greater trochanter and the lateral condyle of the femur and aligned with the direction of the VL fascicles so that the echoes delineating a single fascicle could be tracked throughout the entire range of motion of the knee [15]. A thin echo-absorbent reference strip was fixed to the subject to allow for correction of any probe movement that occurred during the testing. The transducer head were fixed to the subject using a custom-made thermoplastic cast and were taped into place.

Ultrasound images were analysed using Java-based image processing program software (ImageJ, National Institutes of Health, Bethesda, MD, USA). An individual fascicle was tracked throughout the movement and the fascicle length and pennation angle from the deep aponeurosis were recorded for each image. Shortening and lengthening velocities of the fascicle were calculated as the change in length over time during the movement.

Figure 1 showed the step for 30FL and 70FL that were performed in this study. Participants were instructed to stand with their hands holding a weight loaded barbell consisted of $30 \%$ or $70 \%$ 1RM placed on their shoulder, feet shoulder width apart. Participants lunged forward with the dominant foot and lowered the thigh until be parallel with the ground, and then 
returned back to the starting position. Participants were needed to make a big step as during downward position, the knee should not extend beyond the toe. The non-leading foot must not move from its starting position, and the head were constantly faced forward. The trunk was maintained to be straight. Participants were required to perform all the 30FL and 70FL for three trials consisting of three repetitions for each trial for both dominant and non-dominant lower limb.

(a)

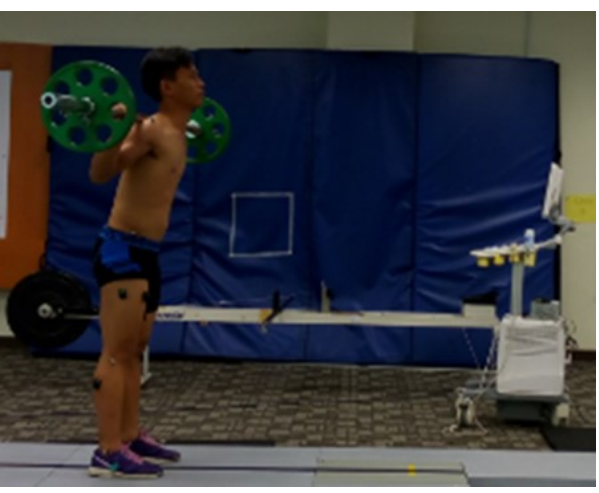

(b)

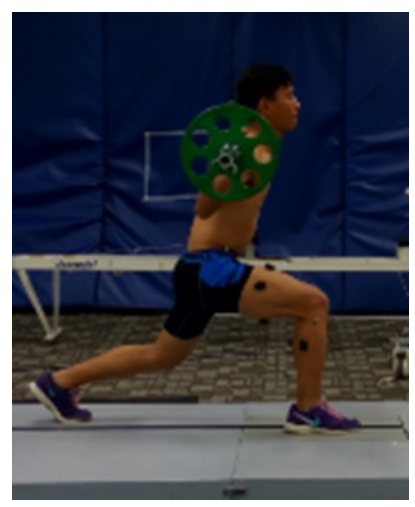

Fig.1. Forward lunge performed in this study

All participants involved in familiarization session in order to make sure all the participants were able to perform all the tests correctly. After familiarization session, participants were tested for their badminton specific forward lunge one repetition maximum (1RM). The 1RM test score were used as a part of dependent variable and as determinant of training loads during this study. Participants were required to refrain from any exercise for at least 48 hours and refrain from alcohol for at least 24 hours prior to the 1RM test and experimental session. To prevent risks of injury incidence during 1RM test, multiple-RM method were implemented as it was recommended to be safer [21].

Both loading protocols were conducted in randomized order to minimise order effects. In order to ensure maximal performance, participants were instructed to "lunge as far as possible and as fast as possible". Fascicle behaviours of the stepping limb (dominant and non-dominant) were assessed during each trial. Comparisons of those variables were made between each lunge protocols and between dominant and non-dominant limbs. All the 
familiarization and data collection sessions were supervised by the researcher with the assistance of appointed trained trainers.

\subsection{Statistical analysis}

Descriptive statistics were used to measure the mean and standard deviation of each physical characteristics and data scores. Repeated measure analysis of multivariances (MANOVA) was used to compare the difference of fascicle behaviours in this study. Statistical significance was accepted at an $\alpha$-level of $p \leq 0.05$. All statistical analyses were conducted using SPSS version 23 (IBM, New York, USA).

\section{RESULTS}

Table 1 showed the physical characteristics of participants involved in this study.

Table 1. Physical Characteristics of Participants

\begin{tabular}{ll}
\hline Variables & Mean \pm SD \\
\hline Age (years) & $21 \pm 0.83$ \\
Body Mass $(\mathrm{kg})$ & $71.00 \pm 1.88$ \\
Body Weight $(\mathrm{N})$ & $696.57 \pm 33.08$ \\
Height $(\mathrm{cm})$ & $171.41 \pm 2.55$ \\
1RM (kg) & $70.97 \pm 6.57$ \\
Relative 1RM (1RM/BM) & $1.00 \pm 0.05$ \\
\hline
\end{tabular}

\subsection{Dominant lower limb}

Analysis on the dominant lower limb showed a significant main effect for all the fascicle behaviour variables: i) maximum FL (FLmax), $F(1,29)=4.462 ; \mathrm{p}<0.05$, ii) minimum FL (FLmin), F(1,29) = 145.000; $\mathrm{p}<0.001$, iii) maximum PA $($ PAmax $), F(1,29)=128.889 ; \mathrm{p}<$ 0.001 , iv) minimum PA (PAmin), $\mathrm{F}(1,29)=376.147 ; \mathrm{p}<0.001$, v) lengthening velocity (LENvel), $\mathrm{F}(1,29)=110.187 ; \mathrm{p}<0.001$ and vi) shortening velocity (SHOvel), F(1,29) = 84.180; $\mathrm{p}<0.001$. 
Table 2. Fascicle Behaviour Data of Dominant Lower Limb during SFL and JFL

\begin{tabular}{lll}
\hline Variables & $30 \mathrm{FL}$ & $70 \mathrm{FL}$ \\
\hline FLmax $(\mathrm{cm})$ & $13.30 \pm 1.40^{\mathrm{b}}$ & $13.38 \pm 1.46^{\mathrm{a}}$ \\
FLmin $(\mathrm{cm})$ & $5.16 \pm 0.61^{\mathrm{b}}$ & $5.31 \pm 0.61^{\mathrm{a}}$ \\
PAmax $\left(^{\circ}\right)$ & $20.67 \pm 1.64^{\mathrm{b}}$ & $21.07 \pm 1.55^{\mathrm{a}}$ \\
PAmin $\left(^{\circ}\right)$ & $10.34 \pm 0.78^{\mathrm{b}}$ & $10.76 \pm 0.76^{\mathrm{a}}$ \\
LENvel $(\%)$ & $18.28 \pm 0.91^{\mathrm{b}}$ & $16.75 \pm 1.70^{\mathrm{a}}$ \\
SHOvel $(\%)$ & $18.13 \pm 0.80^{\mathrm{b}}$ & $17.66 \pm 0.57^{\mathrm{a}}$ \\
\hline
\end{tabular}

${ }^{\mathrm{a}}=$ significantly difference from SFL, $\mathrm{p}<0.05$

${ }^{\mathrm{b}}=$ significantly difference from JFL, $\mathrm{p}<0.05$

Table 2 showed the fascicle behaviour data during the two lunge protocols. Results showed that FLmax, FLmin, PAmax and PAmin were all greater during 70FL compared to 30FL, $\mathrm{p}<$ 0.001. However, lengthening and shortening velocity were found to be greater during $30 \mathrm{FL}$ compared to 70FL, $\mathrm{p}<0.05$.

\subsection{Non-dominant lower limb}

Analysis on non-dominant lower limb showed a significant main effect for all the fascicle behaviour variables: i) maximum FL (FLmax), $F(1,29)=3.222 ; p<0.05$, ii) minimum FL $($ FLmin), $\mathrm{F}(1,29)=123.23 ; \mathrm{p}<0.001$, iii) maximum PA (PAmax), $\mathrm{F}(1,29)=87.65 ; \mathrm{p}<$ 0.001, iv) minimum PA (PAmin), $\mathrm{F}(1,29)=278.34 ; \mathrm{p}<0.001$, v) lengthening velocity (LENvel), $\mathrm{F}(1,29)=92.35 ; \mathrm{p}<0.001$ and vi) shortening velocity (SHOvel), $\mathrm{F}(1,29)=76.548$; $\mathrm{p}<0.001$. 
Table 3. Fascicle Behaviour Data of Non-dominant Lower Limb during SFL and JFL

\begin{tabular}{lll}
\hline Variables & $30 \mathrm{FL}$ & $70 \mathrm{FL}$ \\
\hline FLmax & $12.97 \pm 1.38^{\mathrm{b}}$ & $13.04 \pm 1.45^{\mathrm{a}}$ \\
FLmin & $4.82 \pm 0.60^{\mathrm{b}}$ & $4.97 \pm 0.60^{\mathrm{a}}$ \\
PAmax & $20.06 \pm 1.62^{\mathrm{b}}$ & $20.46 \pm 1.53^{\mathrm{a}}$ \\
PAmin & $9.93 \pm 0.79^{\mathrm{b}}$ & $10.35 \pm 0.78^{\mathrm{a}}$ \\
LENvel & $17.87 \pm 0.92^{\mathrm{b}}$ & $16.34 \pm 1.70^{\mathrm{a}}$ \\
SHOvel & $17.72 \pm 0.83^{\mathrm{b}}$ & $17.25 \pm 0.60^{\mathrm{a}}$ \\
\hline
\end{tabular}

${ }^{\mathrm{a}}=$ significantly difference from SFL, $\mathrm{p}<0.05$

$\mathrm{b}=$ significantly difference from JFL, $\mathrm{p}<0.05$

Table 3 showed the fascicle behaviour data during the two lunge protocols. Results showed that FLmax, FLmin, PAmax and PAmin were all greater during 70FL compared to 30FL, $\mathrm{p}<$ 0.001. However, lengthening and shortening velocity were found to be greater during $30 \mathrm{FL}$ compared to $70 \mathrm{FL}, \mathrm{p}<0.05$.

\section{$3.330 \%$ 1RM forward lunge (dominant versus non-dominant lower limb)}

Analysis on the dominant and non-dominant lower limb during 30FL showed significant main effect for all the fascicle behaviour variables: i) maximum FL (FLmax), F(1,29) = 226.562; $p$ $<0.05$, ii) minimum FL (FLmin), F(1,29) = 247.914; p < 0.001, iii) maximum PA (PAmax), $\mathrm{F}(1,29)=1681.205 ; \mathrm{p}<0.001, \mathrm{iv})$ minimum PA (PAmin), $\mathrm{F}(1,29)=149.432 ; \mathrm{p}<0.001, \mathrm{v})$ lengthening velocity (LENvel), $\mathrm{F}(1,29)=149.432 ; \mathrm{p}<0.001$ and vi) shortening velocity $($ SHOvel), $\mathrm{F}(1,29)=149.432 ; \mathrm{p}<0.001$. Pairwise comparison test showed all the fascicle behaviour variables were greater in the dominant limb compared to non-dominant limb during 30FL.

\section{$3.470 \%$ 1RM forward lunge (dominant versus non-dominant lower limb)}

Analysis on the dominant and non-dominant lower limb during 70FL showed significant main effect for all the fascicle behaviour variables: i) maximum FL (FLmax), F(1,29) = 247.914; p $<0.05$, ii) minimum FL (FLmin), $\mathrm{F}(1,29)=247.914 ; \mathrm{p}<0.001$, iii) maximum PA (PAmax), 
$\mathrm{F}(1,29)=1681.205 ; \mathrm{p}<0.001, \mathrm{iv})$ minimum PA (PAmin), $\mathrm{F}(1,29)=149.432 ; \mathrm{p}<0.001, \mathrm{v})$ lengthening velocity (LENvel), $\mathrm{F}(1,29)=149.432 ; \mathrm{p}<0.001$ and vi) shortening velocity (SHOvel), $\mathrm{F}(1,29)=149.432 ; \mathrm{p}<0.001$. As during the 30FL, pairwise comparison test showed all the fascicle behaviour variables were greater in the dominant limb compared to non-dominant limb during 70FL.

\section{DISCUSSIONS}

The knowledge on fascicle behaviour during movement can indicate the muscle architecture adaptation in the long term. Currently, lack of study has been conducted on the fascicle behaviour during resistance training. The fascicle behaviour during lunge movement was the current study interest as this movement was one of the most specific movement in many sports [22-25].

Maximum fascicle length (FLmax), minimum fascicle length (FLmin), maximum pennation angle (PAmax), minimum pennation angle (PAmin), lengthening velocity (LENvel), and shortening velocity (SHOvel) of vastus lateralis were assessed and compared between loadings used during lunge exercise in this study.

Results in this study showed that FLmax, FLmin, PAmax and PAmin were all greater during 70FL compared to $30 \mathrm{FL}, \mathrm{p}<0.001$. However, lengthening and shortening velocity were found to greater during 30FL compared to $70 \mathrm{FL}, \mathrm{p}<0.05$. These conditions were also seen in both dominant and non-dominant limb.

Results demonstrated that greater loadings $(70 \mathrm{FL})$ caused fascicle to have greater maximum and minimum fascicle lengthening and pennation angle compared to lighter loads (30FL). Until now, less study has been conducted on the effects of loadings on the fascicle behaviour. [15] in their study found loading intensity to affect muscle tendon unit (MTU) behavior. They suggested that the changes were most likely due to the changes in the dynamic stiffness of the tendon and distal aponeurosis, and activation patterns of the muscle despite no differences in peak muscle tendon unit length and fascicle length were found.

This results of this current study showed that force production required more fascicle lengthening and pennation angle. This also showed that rather than velocity, loadings do have greater impact on fascicle behaviour during movement. However, lengthening and shortening 
velocity that were found to be greater during 30FL compared to 70FL demonstrated that the fascicle velocity might be related to the movement kinematics in which ascent and descent phase time were found to be faster during $30 \mathrm{FL}$.

This showed that muscle activity increased as fascicle underwent greater lengthening activity rather than the velocity of movement. However, it should be noted that the greater lengthening and pennation angle was affected by the loadings (70FL) in this study. Thus, the relationship between fascicle behaviour and the muscle activation should be studied more in the future to find the possible acute responses that can affect the adaptations especially in strength and hypertrophy $[1,21-23,26]$.

In this current study, we have found that all the fascicle behaviours responses were shown to be greater in the dominant limb compared to the non-dominant limb. In our knowledge, this is the first study to compare the differences of fascicle behaviour between dominant and non-dominant limb during resistance exercise. The greater fascicle lengthening, velocity and pennation angle in the dominant lower limb could be related to the kinematics, muscle activity and kinetic response that were found to be greater in the dominant limb compared to non-dominant limb. More studies were suggested to be conducted to explore the relationships between all these variables.

\section{CONCLUSIONS}

Findings of this study demonstrate the fascicle behaviours of vastus lateralis were more obvious during forward lunge with greater loads lifted. This thus showed that muscle architecture adaptations are more prone to occur greater loads exercise training.

\section{ACKNOWLEDGEMENTS}

Researchers would like to thank all participants involved in this study.

\section{REFERENCES}

[1] Nadzalan, A.M., et al., Relationship between lower body muscle architecture and lunges performance. Journal of Sports Science and Physical Education, 2016. 5(2): p. 15-23. 
[2] Earp, J.E., et al., Lower-body muscle structure and its role in jump performance during squat, countermovement, and depth drop jumps. The Journal of Strength \& Conditioning Research, 2010. 24(3): p. 722-729.

[3] Abe, T., K. Kumagai, and W.F. Brechue, Fascicle length of leg muscles is greater in sprinters than distance runners. Medicine and Science in Sports and Exercise, 2000. 32(6): p. 1125-1129.

[4] Kumagai, K., et al., Sprint performance is related to muscle fascicle length in male 100-m sprinters. Journal of Applied Physiology, 2000. 88(3): p. 811-816.

[5] Nadzalan, A.M., et al., The effects of step versus jump forward lunge exercise training on muscle architecture among recreational badminton players. World Applied Sciences Journal, 2017. 35(8): p. 1581-1587.

[6. Franchi, M.V., et al., Architectural, functional and molecular responses to concentric and eccentric loading in human skeletal muscle. Acta Physiologica, 2014. 210(3): p. 642-654.

[7] Kim, S.Y., et al., Investigation of supraspinatus muscle architecture following concentric and eccentric training. Journal of Science and Medicine in Sport, 2015. 18(4): p. 378-382.

[8] Weide, G., et al., Medial gastrocnemius muscle growth during adolescence is mediated by increased fascicle diameter rather than by longitudinal fascicle growth. Journal of Anatomy, 2015. 226(6): p. 530-541.

[9] Kawakami, Y., et al., In vivo muscle fibre behaviour during counter-movement exercise in humans reveals a significant role for tendon elasticity. The Journal of Physiology, 2002. 540(2): p. 635-646.

[10]Duclay, J., et al., Behavior of fascicles and the myotendinous junction of human medial gastrocnemius following eccentric strength training. Muscle \& Nerve, 2009. 39(6): p. 819-827.

[11]Hoffren, M., M. Ishikawa, and P.V. Komi, Age-related neuromuscular function during drop jumps. Journal of Applied Physiology, 2007. 103(4): p. 1276-1283.

[12]Ishikawa, M., T. Finni, and P. Komi, Behaviour of vastus lateralis muscle-tendon during high intensity SSC exercises in vivo. Acta Physiologica Scandinavica, 2003. 178(3): p. 205-213. 
[13]Ishikawa, M. and P.V. Komi, Effects of different dropping intensities on fascicle and tendinous tissue behavior during stretch-shortening cycle exercise. Journal of Applied Physiology, 2004. 96(3): p. 848-852.

[14]Franchi, M.V., et al., Architectural, functional and molecular responses to concentric and eccentric loading in human skeletal muscle. Acta Physiologica, 2014. 210(3): p. 642-654.

[15]Earp, J.E., et al., The influence of loading intensity on muscle-tendon unit behavior during maximal knee extensor stretch shortening cycle exercise. European Journal of Applied Physiology, 2014. 114(1): p. 59-69.

[16]Mian, O.S., et al., Gastrocnemius muscle-tendon behaviour during walking in young and older adults. Acta Physiologica, 2007. 189(1): p. 57-65.

[17]Cronin, N.J. and T. Finni, Treadmill versus overground and barefoot versus shod comparisons of triceps surae fascicle behaviour in human walking and running. Gait \& Posture, 2013. 38(3): p. 528-533.

[18]Lai, A., et al., In vivo behavior of the human soleus muscle with increasing walking and running speeds. Journal of Applied Physiology, 2015. 118(10): p. 1266-1275.

[19]Panizzolo, F.A., et al., Soleus fascicle length changes are conserved between young and old adults at their preferred walking speed. Gait \& Posture, 2013. 38(4): p. 764-769.

[20]Jönhagen, S., K. Halvorsen, and D.L. Benoit, Muscle activation and length changes during two lunge exercises: implications for rehabilitation. Scandinavian Journal of Medicine \& Science in Sports, 2009. 19(4): p. 561-568.

[21]Baechle, T.R. and R.W. Earle, Essentials of strength training and conditioning. Vol. 7. 2008: Human Kinetics Champaign, IL.

[22]Kuntze, G., N. Mansfield, and W. Sellers, A biomechanical analysis of common lunge tasks in badminton. Journal of Sports Sciences, 2010. 28(2): p. 183-191.

[23]Bober, T., et al., Original research papers. Kinematic characterisation of the lunge and the fleche in epee fencing: two case studies. Polish Journal of Sport and Tourism, 2016. 23(4): p. 181-185.

[24] Sinclair, J., et al., Influence of minimalist footwear on knee and ankle loads during the squash lunge. Movement \& Sport Sciences, 2016(1): p. 77-84. 
[25]Nadzalan, A.M., et al., Muscle activation analysis of step and jump forward lunge among badminton players. Journal of Engineering and Science Research, 2017. 1(2): p. 60-65.

[26]Nicholson, G., et al., Do the acute biochemical and neuromuscular responses justify the classification of strength-and hypertrophy-type resistance exercise? The Journal of Strength \& Conditioning Research, 2014. 28(11): p. 3188-3199.

\section{How to cite this article:}

Nadzalan A. M., Mohamad N. I, Low J. F. L, Ahmad R. and Waqqash E. Fascicle Behaviour Analysis during Forward Lunge Exercise: The Comparisons between Training Loads. J. Fundam. Appl. Sci., 2017, 9(6S), 1090-1101. 